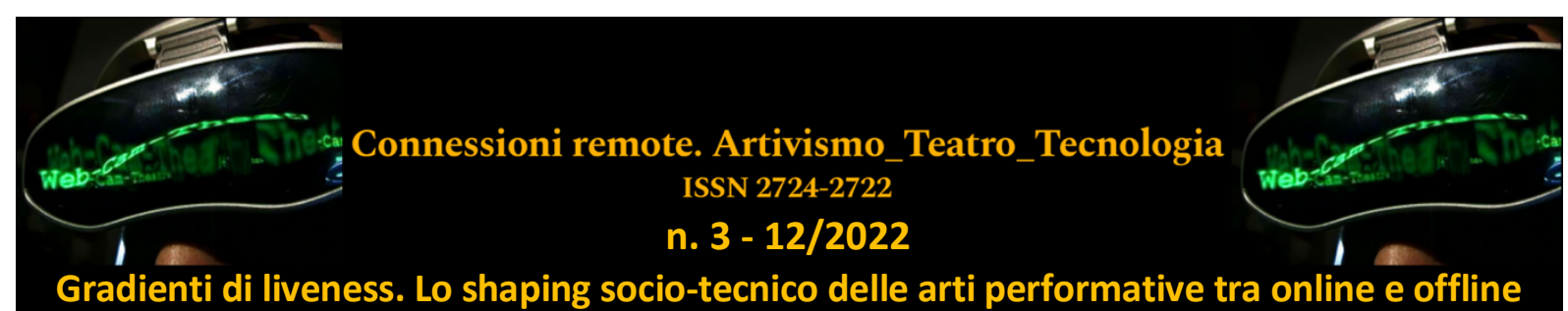

\title{
Gradienti di liveness. Lo shaping socio-tecnico delle arti performative tra online e offline Introduzione
}

\author{
Laura Gemini \\ Università degli Studi di Urbino Carlo Bo \\ Stefano Brilli \\ Università degli Studi di Urbino Carlo Bo
}

\section{La mediatizzazione come frame per la liveness}

A ormai due anni dall'inizio della pandemia e dalle conseguenze prodotte a livello del sistema di produzione e consumo culturale, è possibile affermare con una certa sicurezza, non tanto di essere entrati in una nuova fase di sviluppo del processo di mediatizzazione del sociale, quanto piuttosto di trovarci in una situazione inedita di consapevolezza diffusa - anche se non per questo a-problematica - sui suoi effetti ${ }^{1}$.

Affrontare un tema come la liveness attraverso la prospettiva di osservazione della mediatizzazione, significa prima di tutto prendere una posizione teorica e di analisi della comunicazione dal vivo come processo caratterizzato dalle possibilità di articolazione della presenza ${ }^{2}$ - delle condizioni di condivisione spazio-temporale dei partecipanti - che dipende dalle dimensioni socio-tecniche prodotte dal sistema dei media e da internet. È

\footnotetext{
${ }^{1}$ Cfr. S. Livingstone, P. Lunt, Mediatization: an emerging paradigm for media and communication research, in $\mathrm{K}$. Lundby (a cura di), Mediatization of Communication: Handbooks of Communication Science vol. 21, De Gruyter Mouton, Berlin 2014, pp. 703-723; G. Boccia Artieri, Mediatizzazione e Network Society: un programma di ricerca, in «Sociologia della Comunicazione», n. 50, 2015, pp. 62-69; N. Couldry, A. Hepp, The mediated construction of reality, Polity Press, Cambridge 2017.

${ }^{2}$ Cfr. L. Gemini, S. Brilli, F. Giuliani, II dispositivo teatrale alla prova del Covid-19. Mediatizzazione, liveness e pubblici, in «Mediascapes Journal», n. 15, 2020, pp. 44-53.
} 
pertanto in questo senso che la liveness può essere osservata alla luce del processo globale di mediatizzazione che permea tutti gli ambiti della vita sociale.

Può dunque valere la pena precisare in questa sede come il termine "mediatizzazione" rimandi a un concetto utilizzato nelle scienze sociali e negli studi sui media per comprendere le trasformazioni socio-tecnologiche e come le modalità di mediazione tecno-comunicativa penetrano e trasformano un numero sempre maggiore di domini sociali: dalla politica, all'economia, dalle forme educative, al campo della medicina, ai contesti dell'informazione fino, naturalmente, al campo culturale e artistico. La mediatizzazione va pertanto considerata come un meta-processo ${ }^{3}-$ al pari di concetti come globalizzazione, individualizzazione, modernizzazione - che coinvolge diversi campi e si manifesta in maniere differenti.

La letteratura dedicata alla ricerca su questo tema mette inoltre in evidenza come il mutamento socio-tecnico non vada considerato in chiave deterministica ma debba piuttosto tenere conto, da un lato, delle pratiche che riguardano il modo con cui enti e organizzazioni influiscono nel progettare i media e le tecnologie con le norme di utilizzo e, dall'altro lato, delle agency ${ }^{4}$ individuali e collettive che "piegano" il significato dei media e delle tecnologie nei contesti d'uso quotidiani. Pensiamo, a livello collettivo ad esempio, al modo in cui movimenti e gruppi immaginano nuovi utilizzi come nel caso del movimento hacker o alle comunità dei maker, oppure, al ruolo giocato dagli artisti o da certi movimenti nel campo della sperimentazione mediale e tecnologica ${ }^{5}$. La mediatizzazione è dunque la chiave per comprendere le dinamiche di modellamento da parte delle tecnologie mediali che forniscono formati e frame all'esperienza. Basti pensare a come i for-

\footnotetext{
${ }^{3}$ F. Krotz F., Mediatization: A Concept with Which to Grasp Media and Societal Change, in K. Lundby (a cura di), Mediatization: Concept, Changes, Consequences, Peter Lang, New York 2009, pp. 21-40.

${ }^{4}$ Nell'ottica di Judith Butler il concetto di "agency" (agentività) riguarda una concezione dell'agire che deve essere considerata a partire dalle condizioni da cui emerge, da cui è indotto, supportato o ostacolato e che sono di ordine culturale, politico, socio-economico. Si tratta dunque di un agire strutturato ma che costituisce allo stesso tempo una forma di agire che può cambiare la sua stessa strutturazione. Cfr. F. Zappino in J. Butler, L'alleanza dei corpi. Note per una teoria performativa dell'azione collettiva, nottetempo, Milano 2017. In questo senso il concetto di agency si rivela particolarmente pertinente all'analisi critica della mediatizzazione, poiché riesce a tenere insieme i constraints tecno-mediali con le pratiche di utilizzo, e conseguentemente si dimostra particolarmente utile all'analisi dei processi di mediatizzazione artistica e performativa che riguardano la liveness.

${ }^{5}$ Si veda, ad esempio, l'esperienza del tecno-artista Giacomo Verde basata sull'uso delle tecnologie per la realizzazione di progetti artistici e sociali e, su questa linea, il campo di azione dell'artivismo digitale. Cfr. AA.VV., L'artivismo: forme, esperienze, pratiche e teorie, in «Connessioni remote. Artivismo_Teatro_Tecnologie», vol. 2, n. 2, 2020.
} 
mati televisivi, cinematografici, video e dei media digitali abbiano influito sulla trasformazione delle arti performative ${ }^{6}$ e all'affermazione di una semantica della performance che si è appropriata delle logiche tecno-mediali nei termini del frammento, della serialità, del cut-up così come dell'intermedialità, della trans-medialità, della shareability, insieme alle idee dei pubblici come audience partecipative e come community ${ }^{7}$.

Se dunque il processo di mediatizzazione riguarda la penetrazione delle logiche mediali nei contesti della vita quotidiana, la mediatizzazione digitale $e^{8}$ - nella sua integrazione di web, social media, mobile, sistemi di IA, ecc. - comporta ulteriori cambiamenti che riguardano, fra gli altri, la riarticolazione del rapporto fra comunicazione interpersonale e di massa, le condizioni spazio-temporali dell'interazione sociale e il senso di prossimità (e distanza) attraverso le tecnologie di rete. In questo senso internet è diventata un contesto normalizzato dell'agire dove il vissuto dell'ambiente quotidiano passa attraverso la pervasività dei media di connessione e le infrastrutture che mettono a punto un diverso rapporto con l'apprendimento, il lavoro, il consumo, l'informazione e l'intrattenimento. La mediatizzazione digitale ha inoltre favorito il processo di platformization del sociale ${ }^{9}$, ovvero la tendenza a regolare un vasto numero di interazioni sociali ma anche le dinamiche di produzione, distribuzione e fruizione di contenuti, attraverso strumenti di disintermediazio$n \mathrm{e}^{10}$ - le piattaforme appunto - che operano anche in maniera sempre più evidente nel campo della produzione e del consumo dei contenuti artistici e culturali ${ }^{11}$.

Sono questi i presupposti su cui, crediamo, è necessario ripartire per affrontare il tema della liveness attraverso gli strumenti teorici e metodologici che provengono dagli studi sui media e per osservarla come quella particolare condizione della comunicazione dal vivo che si realizza nei contesti mediatizzati.

\footnotetext{
${ }^{6}$ Cfr. A.M. Monteverdi, Nuovi media, nuovo teatro. Teorie e pratiche tra teatro e digitalità, FrancoAngeli, Milano 2011.

${ }^{7}$ L. Gemini, Post-Novecento e mediatizzazione. Appunti mediologici sulle arti performative, in C. Tafuri, C. Beronio (a cura di), Ivrea Cinquanta. Mezzo secolo di nuovo teatro in Italia 1967-2017, Akropolis Libri, Genova 2018, pp. 216-223.

${ }^{8}$ Cfr. N. Couldry, A. Hepp, The Mediated Construction of Reality: Society, Culture, Mediatization, Polity Press, Cambridge 2017; G. Boccia Artieri, L. Gemini, F. Pasquali, S. Carlo, M. Farci, M. Pedroni, Fenomenologia dei Social Network. Presenza, relazioni e consumi mediali degli italiani online, Guerini, Milano 2017.

${ }^{9}$ J. Van Dijck, T. Poell, M. Waal, Platform Society. Valori pubblici e società connessa, Guerini, Milano 2019.

${ }^{10}$ Cfr. G. Boccia Artieri, 2015, op. cit.

${ }^{11}$ T. Poell, D. Nieborg, B.E. Duffy, Platforms and Cultural Production, Polity Press, Cambridge 2021.
} 


\section{Liveness e mediatizzazione della presenza}

L'analisi dei processi comunicativi dal vivo nei contesti mediatizzati può contare su un'autorevole tradizione di studi che da Walter Benjamin passa per autori che si sono occupati della dimensione live nei contesti mass mediali come Paddy Scannell ${ }^{12}$ e John B. Thompson ${ }^{13}$ per arrivare al celebre lavoro di Philip Auslander ${ }^{14}$ che, ponendo la liveness come condizione fenomenologica più che come caratteristica insita nel medium, ha aperto un ramo di studi che da oltre vent'anni si interroga sulle condizioni dell'emersione di questa particolare esperienza.

Com'è noto, inoltre, la riflessione sulla liveness ha subito una significativa accelerazione con la crisi pandemica ${ }^{15}$ che ha reso ancor più evidente la centralità del concetto a partire dalla mancanza forzata delle occasioni dal vivo basate sull'hic et nunc tradizionalmente inteso.

La copertura mediale e online di eventi, performance, progetti artistici, teatrali, musicali, ecc. ha permesso di proporre e fruire di diversi contenuti culturali ${ }^{16}$, di non fermare l'espressività artistica e stimolare una forma a distanza della relazione performativa. Ma ha soprattutto permesso, e permette tuttora, di osservare come un'accezione più complessa e "mobile" del concetto di liveness consenta di esaminare la riarticolazione del senso di simultaneità della presenza sulla base del processo di mediatizzazione che, come si è detto, riformula il rapporto fra interazione (tipica della comunicazione interpersonale) e comunicazione mediata.

Negli ambienti online, ad esempio, si può osservare la realizzazione di quella particolare declinazione della liveness, che Auslander definisce "digital liveness" che non scaturisce solo dalle proprietà degli ambienti digitali o dalla pura costruzione del pubblico, ma riguarda «[...] a specific relation between self and other, a particular way of "being

\footnotetext{
12 P. Scannell, Radio, TV and modern life, Blackwell Publishers, Oxford 1996.

13 J.B. Thompson, Mezzi di comunicazione e modernità. Una teoria sociale dei media, il Mulino, Bologna 1998.

${ }^{14}$ P. Auslander Liveness: Performance in a Mediatized Culture, Routledge, London and New York 1999.

${ }^{15}$ Cfr. L. Gemini, Antidoto liveness. La performance dal vivo durante il Covid-19, in "Sociologia della Comunicazione», n. 60, 2020, pp. 104-118; K. Capece, P. Scorese (a cura di), Pandemic performance: resilience, liveness, and protest in quarantine times, Routledge, New York, 2021; L. Gemini, S. Brilli, F. Giuliani, Liveness e pandemia. Percorsi di ricerca sulla mediatizzazione del teatro a teatri chiusi, in G. Boccia Artieri, M. Farci (a cura di), Shockdown. Media, cultura, comunicazione e ricerca nella pandemia, Meltemi, Milano 2021, pp. 141-160.

${ }^{16}$ M. Banks, The work of culture and C-19, in «European Journal of Cultural Studies», vol. 23, n. 4, 2020, pp. 648-654.
} 
involved with something". The experience of liveness results from our conscious act of grasping virtual entities as live in response to the claims they make on us». ${ }^{17}$

Questo specifico framework fenomenologico è stato ampliato da altre importanti analisi. Come ad esempio quella di Martin Barker ${ }^{18}$ che, a partire da indagini empiriche sulle audience, identifica la liveness come esperienza composita, cui partecipano almeno sette componenti: 1) la compresenza fisica fra performer e performance, 2) la simultaneità, 3) il senso dell'engagement non mediato, 4) il senso della località con l'esperienza, 5) il senso dell'interazione con il performer, 6) il senso con l'interazione con gli altri membri del pubblico e 7) la coscienza di partecipare a un evento unico. Oppure quella di Paul Sanden ${ }^{19}$, che lavorando sulla mediatizzazione dei concerti, traccia un'altra classificazione riguardante le modalità di riconoscimento della liveness nella performance musicale, distinguendo: temporal liveness quando si è presenti nel momento dell'emissione musicale primaria, spatial liveness quando si condivide lo spazio di questa emissione, liveness of fidelity che riguarda la valutazione di fedeltà alla performance iniziale, liveness of spontaneity laddove l'attenzione è posta sulla spontaneità e imprevedibilità della prestazione umana, corporeal liveness relativa al grado di connessione percepita con il corpo acustico dell'emissione, interactive liveness derivante dall'interazione fra performer e fra performer e pubblico, virtual liveness quando la percezione della liveness dipende dall'interazione con un corpo interamente mediatizzato.

Questi aspetti non si realizzano quindi esclusivamente nel contesto della comunicazione dal vivo ontologicamente intesa ${ }^{20}$, per cui è possibile intendere la liveness come un dispositivo che produce una posizione del soggetto, che dipende da discorsi e frame istituzionali, e che si costruisce relazionalmente con altri membri del pubblico, contesti d'uso e tecnologie. Vi è cioè una dimensione non soltanto esperienziale della liveness, ma anche una sua componente discorsiva che la rende uno strumento di categorizzazio-

\footnotetext{
${ }^{17}$ P. Auslander P., Digital Liveness. A Historico-Philosophical Perspective, in «PAJ. A Journal of Performance and Art», n. 102, p. 10.

${ }^{18}$ M. Barker, "Live at a cinema near you": How audiences respond to digital streaming of the arts, in J. Radbourne, $\mathrm{H}$. Glow, K. Johanson (a cura di), The audience experience: A critical analysis of audiences in the performing arts, Intellect, Bristol 2013, pp. 15-34.

${ }^{19}$ P. Sanden, Liveness in modern music: musicians, technology, and the perception of performance, Routledge, London and New York 2013.

${ }^{20}$ L. Gemini, Liveness: le logiche mediali nella comunicazione dal vivo, in «Sociologia della Comunicazione», $\mathrm{n}$. 51, 2016, pp. 43-63.
} 
ne e distinzione. Già Sarah Thornton nel suo seminale studio sulle club culture del $1995^{21}$ evidenziava come l'aver partecipato al "vero evento musicale dal vivo" fosse impiegato come un indicatore dello status subculturale degli adepti. Se quindi gli studi teorici ed empirici degli ultimi vent'anni tendono ad "aprire" la liveness, riconoscendo la diversità dei significati, delle condizioni di realizzazione, dei piaceri che gli spettatori vi associano, dall'altra parte vi è una pressione discorsiva che mira a binarizzare la distinzione live/non-live, alimentata in particolare dai processi di valorizzazione economica e simbolica di eventi e tecnologie che sarebbero capaci di veicolare il "vero dal-vivo". Osservare la liveness in termini di "gradienti" 22 vuole quindi essere un invito a tenere lo sguardo sia sulla componente multidimensionale e graduale del fenomeno, del suo modo di realizzarsi in forme non dicotomiche, ma anche sul fatto che esistono forze che rendono discreti questi gradienti, e che entrambi questi fattori meritano l'attenzione della ricerca.

Su queste basi diviene interessante capire quali dispositivi di liveness siano attivi nella sperimentazione performativa e artistica a partire dalle possibili riarticolazioni del rapporto fra qui e ora: sia a livello creativo, poetico e drammaturgico in ottica intermediale e transmediale, sia sul piano della messa a punto di dinamiche relazionali e di gestione della presenza mediatizzata dei partecipanti che tengano conto delle nuove forme della tele-presenza, della corporeità e dell'esperienza aptica negli ambienti digitali ${ }^{23}$.

Questo tipo di analisi acquisisce oggi un ulteriore elemento di interesse in base a quello che autori come Benjamin Haber ${ }^{24}$ definiscono un ephemeral turn nei media digitali. Tale "svolta" riguarda il passaggio dalla permanenza dei contenuti come condizione predefinita 25 alle molteplici temporalità di story e live stream, che apre nuove possibilità performative alle piattaforme online e ai social media che necessitano ancora di essere adeguatamente esplorate. L'accelerazione che si è verificata con il lockdown, dovuta alla necessità per gli artisti e le organizzazioni teatrali di arginare, almeno in parte, la chiusura dei teatri e delle manifesta-

\footnotetext{
${ }^{21}$ S. Thornton, Club Cultures: Music, Media and Subcultural Capital, Routledge, London and New York 1995.

${ }^{22}$ L. Gemini, 2016, op. cit.

${ }^{23}$ L. Gemini, S. Brilli, F. Giuliani, op. cit.

${ }^{24}$ B. Haber, The digital ephemeral turn: queer theory, privacy, and the temporality of risk, in Media, Culture \& Society, vol. 41, n. 8, 2019, pp. 1069-1087.

${ }^{25}$ Cfr. d. boyd, Social Network Sites as Networked Publics: Affordances, Dynamics, and Implications, in Z. Papacharissi (a cura di), A networked Self: Identity, Community, and Culture on Social Network Sites, Routledge, London and New York 2010, pp. 39-58.
} 
zioni dal vivo, segna un passo avanti importante nella ricerca sulla liveness e sui suoi diversi gradienti che la sperimentazione Novecentesca ha avviato e che una parte considerevole del mondo culturale e artistico contemporaneo continua ad esplorare.

Il terzo numero di Connessioni Remote intende fornire un nuovo contributo al dibattito creatosi attorno al concetto di liveness. L'obiettivo principale consiste nel comprendere come questa particolare dimensione della comunicazione si sincronizzi con le trasformazioni dell'ecosistema mediale e delle tecnologie digitali ampliando la sua fenomenologia. Più precisamente quello che interessa indagare sono le arti performative intese come ambiti privilegiati per la sperimentazione delle affordance tecno-mediali live e "attivatori" dei processi che stanno alla base della complessificazione della liveness.

Nel contributo Archeologie della presenza: Digital liveness, vanish liveness e la mediatizzazione della presenza, Vincenzo Del Gaudio affronta la liveness e i suoi gradienti in ottica mediologica mettendo in relazione l'approccio mediarcheologico con quello della mediatizzazione del sociale e che, al di là delle posizioni essenzialiste, riguarda anche il campo del teatro e delle arti performative. A partire dall'esempio del Théâtrophone, medium ottocentesco per la comunicazione a distanza da considerare come una delle prime forme della mediatizzazione dell'hic et nunc e della presenza, l'articolo osserva il telefono analogico e digitale nei termini della liveness riportando alcuni esempi di rimediazione teatrale del dispositivo telefonico volte a mostrare come alcune esperienze teatrali contemporanee possano essere lette alla luce delle tecnologie teatrali-telefoniche del passato. Un processo che coinvolge i dispositivi di telefonia mobile e le piattaforme di social media - come nel caso delle performance su Instagram di Nicola Vicidomini riportate nell'articolo - e che permettono all'Autore di introdurre la nozione di "vanish liveness", ovvero quell'idea (e pratica) di una particolare forma della liveness digitale, che rimedia l'originale impermanenza della forma dal vivo secondo le logiche dell'online.

Le molteplici temporalità del livecasting sono al centro del contributo di Sarah Byrne dal titolo Livecasting without the live: The multiple temporalities of National Theatre at Home. Sin dal lancio di NT Live nel 2009, il National Theatre è sempre stato tra i principali punti di riferimento (sia nel Regno Unito che nel resto del mondo) per quanto riguarda la trasmissione digitale live di eventi teatrali. Nel suo articolo Byrne analizza i 
punti di continuità e divergenza tra NT Live e il programma di contenuti streaming dell'NT at Home con cui il National Theatre ha risposto al lockdown. Attingendo alla recente letteratura sul livecasting ed esaminando il corpus para-testuale prodotto attorno all'NT at Home, l'autrice propone una profonda riflessione sui gradienti temporali, spaziali e relazionali dell'iniziativa: sebbene I'NT at Home non si basi sulla trasmissione in simultanea come l'NT Live, il senso dell'evento non è del tutto dissolto, ma ricostruito attraverso la partecipazione del pubblico a un "ora" stratificato, composto dalla condivisione dell'offerta performativa e del comune presente pandemico.

L'impatto della pandemia sullo spettacolo dal vivo, sia sul piano produttivo sia sul piano della fruizione dei pubblici, ha generato un contesto inedito nel solco del quale, un campo specifico come quello dell'opera lirica ha potenziato la sua originaria vocazione alla multimodalità e sperimentato un'interessante "fenomenologia dello streaming", trasformando la condizione di liveness del teatro musicale. Su questo complesso presupposto di partenza Laura Pernice nell'articolo Streammare l'opera lirica. Gli esperimenti di digital liveness del teatro musicale contemporaneo, analizza una selezione dei più recenti e significativi casi di opere in streaming per riflettere sui gradienti di digital liveness dell'opera in video, sia in diretta sia in differita secondo diversi livelli d'intensità dello streaming, mettendo in relazione i presupposti epistemologici della liveness con le diverse caratteristiche della spettatorialità richieste dallo spettacolo lirico e dalla sua necessaria ridefinizione nei formati in streaming.

In Nft e Concerti: l'industria musicale tra liveness, sperimentazioni e alternative future, Alfonso Amendola e Michelle Grillo indagano la mediatizzazione della liveness nel campo dei concerti dal vivo, prendendo in esame il persistere della musica live durante la pandemia e guardando in particolare ai più recenti sviluppi dei NFT (Non-Fungible Token). Gli Autori esplorano come il tentativo di mantenere viva la relazione tra artisti e pubblico tramite forme concertistiche digitalizzate abbia attuato una ricombinazione di codici mediali che ha intensificato i processi di multimedialità, transmedialità e intermedialità nel settore musicale. Attraverso l'analisi di casi nazionali e internazionali, l'articolo descrive l'evolversi in senso non dicotomico e non deterministico dell'articolazione fra online e offline nella musica dal vivo. La tecnologia NFT, in questo senso, mostra 
un'applicazione del digitale che anziché amplificare la deriva della replicabilità - considerata talvolta come un "destino" delle affordance della rete - ricostruisce artificialmente una scarsità dell'accesso e della presenza, principi che a loro volta costituiscono attivatori potenziali della liveness.

Nella prospettiva di un'apertura del concetto di liveness a pratiche performative eterogenee, il contributo di Sara Pastore, dal titolo Performare lo squat. I luoghi culturali occupati come esercizio di liveness e democrazia tratta lo squatting culturale - relativo alla creazione di centri sociali che operano in campo culturale sul territorio italiano come fenomeno che innestandosi nel campo della public art, rimanda a delle esperienze che si collocano nel crocevia tra pratica artistica e movimento di protesta e che pertanto possono essere trattate in termini di liveness. Le esperienze di cultural squatting, nella lettura di Sara Pastore, operano infatti sì sui livelli della co-presenza di performer e audience, della simultaneità di produzione e ricezione e sull'istantaneità ma si tratta allo stesso tempo di realtà che operano in contesti mediatizzati e che si avvalgono della possibilità di connessione tra partecipanti e del senso di co-presenza esperibile online.

II saggio di Francesco Melchiorri, II Cinema VR come esperienza immersiva archischermica, espande l'esplorazione della liveness nel campo degli ambienti mediali immersivi, e del Cinema VR nello specifico. Prendendo le mosse da come l'immersività implichi un ruolo attivo e partecipe dell'osservatore, Melchiorri avanza la necessità di approntare strumenti teorici appropriati per descrivere il rapporto fra la corporeità dell'immagine VR e la corporeità del soggetto dell'esperienza. Fra questi due poli si creerebbe infatti una reciprocità più vicina alla condizione della liveness digitale che a quella del canonico rapporto spettatore-schermo. Per analizzare tale relazione l'Autore ricorre ai concetti di "im-medialità" di Yves Citton e di "archi-schermo" di Mauro Carbone. Quest'ultimo, in particolare, rappresenta per Melchiorri una chiave teorica per approcciarsi ai più recenti sviluppi dei media immersivi. A tal fine viene presentato come caso di studio l'installazione in realtà virtuale CARNE y ARENA presentata al Festival di Cannes del 2017 dal noto regista messicano Alejandro González Iñárritu.

In II teatro delle piccole cose: Quiet Ensemble, Maia Giacobbe Borelli ci porta a riflettere sulla molteplicità e l'apertura dei gradienti di liveness attraverso il lavoro della 
"factory crossmediale" Quiet Ensemble. II progetto, formato dal duo di artisti Bernardo Vercelli e Fabio Di Salvo, percorre da più di un decennio una peculiare traiettoria di ricerca lavorando nell'intersezione tra tecnologia e natura, fisicità e immaterialità, sonoro e visivo, registrazione e dal vivo. L'ampio uso sulla scena di agenti tecnologici e viventi non-umani amplifica i connotati della liveness ben oltre la canonica disposizione dei ruoli fra performer e pubblico, nonché oltre la simultaneità come nucleo valoriale dell'esperienza dal vivo. Si tratta invece di una liveness che pone al centro l'indeterminatezza del vivente, il tempo dilatato della trasformazione graduale e la disappearance. Quest'ultima è qui intesa come resistenza alla riproducibilità, ma anche come ottica rivolta alla materia vivente invisibile nella quotidianità e che quindi, nelle parole dell'Autrice, consente "l'emersione di un teatro del dettaglio che mette in scena il rivelarsi a noi dello spazio organico e naturale».

Lo statuto performativo degli agenti non-umani, e le implicazioni della loro agentività sulla liveness, sono anche al centro dell'articolo di Federica Patti dal titolo La performatività posthuman. Partendo dal repertorio teorico del postumanesimo e del femminismo neo-materialista, attingendo in particolare al pensiero di Karen Barad, il contributo analizza numerose esperienze recenti di live media performance che mettono in scena, e a tema, l'interconnessione fra performer umani e agenti tecnologici. Lo sguardo e l'esperienza curatoriale di Patti ci conducono quindi in un percorso transdisciplinare (nel metodo e negli oggetti selezionati) tra teorie e pratiche della scena tecnologica. I lavori di Fronte Vacuo, fuse*, LaTurbo Avedon, Kamilia Kard, Massiel Leza, Hiroaki Umeda, Rhizomatiks, Choy Ka Fai, Alexander Whitley e Holly Herndon vengono quindi esposti come casi di relazionalità con la tecnosfera, ma anche usati come particelle fra loro entangled di una trama artistica e culturale che sfugge a sintesi unitarie.

Chiude la parte monografica del fascicolo l'articolo di Alessandro Anglani Sephirot-Il Gioco: passaggio tra rappresentazione e simulazione. Nel contributo Anglani presenta il sistema Sephirot-I/ Gioco(C per la creazione di performance interattive da lui sviluppato a partire dal 2019. Questo caso di studio permette all'Autore di innescare una riflessione sul rapporto fra gioco e teatro e sullo stato attuale delle drammaturgie ipertestuali, nonché del modo in cui esse interpellano una concezione di liveness allargata. Questa ri- 
guarda sia la connotazione più vicina ai performance e media studies, come senso della presenza dell'evento performativo, sia il significato proveniente dall'informatica, quale modalità di distribuzione nel tempo delle operazioni tra sistemi di elaborazione concorrenti. La condivisione del tempo della performance emerge quindi come un problema complesso, acuito dalla componente interattiva che amplifica la ripartizione dei ruoli, e dilata i tempi e gli spazi dell'opera/processo.

I nove articoli che compongono il numero riescono a mostrare le ampie diramazioni della questione liveness, rilevandone la pluralità di significati, componenti e ambiti di ricerca: teatro, concerti, danza e opera lirica, ma anche realtà virtuale, performance digitale, drammaturgie interattive, gaming, social media, intelligenze creative digitali, spazi politici e movimenti di protesta. In uno stato in cui la mediatizzazione è integrale alla produzione del sociale, fare ricerca sulle aspettative e sulle condizioni di emergenza della liveness vuol dire indagare mediologicamente la costruzione della presenza. Se il nucleo primigenio del dibattito interrogava un tipo specifico e privilegiato di presenza - quella della performance - è ora chiaro che la liveness è uno strumento d'indagine versatile, un apparato concettuale aperto e generativo utile a esaminare il senso della connessione nella contemporaneità.

\section{Riferimenti bibliografici}

AA.VV., L'artivismo: forme, esperienze, pratiche e teorie, in "Connessioni remote. Artivismo_Teatro_Tecnologie»,vol. 2, n. 2, 2020.

P. Auslander Liveness: Performance in a Mediatized Culture, Routledge, London and New York 1999.

P. Auslander, Digital Liveness: A Historico-Philosophical Perspective, in «Journal of Performance and Art», vol. 34, n. 3, 2012, pp. 3-11.

M. Banks, The work of culture and C-19, in «European Journal of Cultural Studies», vol. 23, n. 4, 2020, pp. 648-654.

M. Barker, "Live at a cinema near you": How audiences respond to digital streaming of the arts, in J. Radbourne, H. Glow, K. Johanson (a cura di), The audience experience: A critical analysis of audiences in the performing arts, Intellect, Bristol 2013, pp. 15-34. 
G. Boccia Artieri, Mediatizzazione e Network Society: un programma di ricerca, in "Sociologia della Comunicazione», n. 50, 2015, pp. 62-69.

G. Boccia Artieri, L. Gemini, F. Pasquali, S. Carlo, M. Farci, M. Pedroni, Fenomenologia dei Social Network. Presenza, relazioni e consumi mediali degli italiani online, Guerini, Milano 2017.

d. boyd, Social Network Sites as Networked Publics: Affordances, Dynamics, and Implications, in Z. Papacharissi (a cura di), A networked Self: Identity, Community, and Culture on Social Network Sites, Routledge, London and New York 2010, pp. 39-58.

J. Butler, L'alleanza dei corpi. Note per una teoria performativa dell'azione collettiva, nottetempo, Milano 2017.

K. Capece, P. Scorese (a cura di), Pandemic performance: resilience, liveness, and protest in quarantine times, Routledge, New York, 2021.

N. Couldry, A. Hepp, The mediated construction of reality, Polity Press, Cambridge 2017.

L. Gemini, Liveness: le logiche mediali nella comunicazione dal vivo, in "Sociologia della Comunicazione», n. 51, 2016, pp. 43-63.

L. Gemini, Post-Novecento e mediatizzazione. Appunti mediologici sulle arti performative, in C. Tafuri, C. Beronio (a cura di), Ivrea Cinquanta. Mezzo secolo di nuovo teatro in Italia 1967-2017, Akropolis Libri, Genova 2018, pp. 216-223.

L. Gemini, Antidoto liveness. La performance dal vivo durante il Covid-19, in «Sociologia della Comunicazione», n. 60, 2020, pp. 104-118.

L. Gemini, S. Brilli, F. Giuliani, Il dispositivo teatrale alla prova del Covid-19. Mediatizzazione, liveness e pubblici, in «Mediascapes Journal», n. 15, 2020, pp. 44-53.

L. Gemini, S. Brilli, F. Giuliani, Liveness e pandemia. Percorsi di ricerca sulla mediatizzazione del teatro a teatri chiusi, in G. Boccia Artieri, M. Farci (a cura di), Shockdown. Media, cultura, comunicazione e ricerca nella pandemia, Meltemi, Milano 2021, pp. 141-160.

B. Haber, The digital ephemeral turn: queer theory, privacy, and the temporality of risk, in Media, Culture \& Society, vol. 41, n. 8, 2019, pp. 1069-1087.

F. Krotz F., Mediatization: A Concept with Which to Grasp Media and Societal Change, in K. Lundby (a cura di), Mediatization: Concept, Changes, Consequences, Peter Lang, New York 2009, pp. 21-40.

S. Livingstone, P. Lunt, Mediatization: an emerging paradigm for media and communication research, in K. Lundby (a cura di), Mediatization of Communication: Handbooks of Communication Science vol. 21, De Gruyter Mouton, Berlin 2014, pp. 703-723.

A.M. Monteverdi, Nuovi media, nuovo teatro. Teorie e pratiche tra teatro e digitalità, FrancoAngeli, Milano 2011. 
T. Poell, D. Nieborg, B.E. Duffy, Platforms and Cultural Production, Polity Press, Cambridge 2021.

P. Sanden, Liveness in modern music: musicians, technology, and the perception of performance, Routledge, London and New York 2013.

P. Scannell, Radio, TV and modern life, Blackwell Publishers, Oxford 1996.

J.B. Thompson, Mezzi di comunicazione e modernità. Una teoria sociale dei media, il Mulino, Bologna 1998.

S. Thornton, Club Cultures: Music, Media and Subcultural Capital, Routledge, London and New York 1995.

J. Van Dijck, T. Poell, M. Waal, Platform Society. Valori pubblici e società connessa, Guerini, Milano 2019. 